\title{
A Statistical Model of Right Ventricle in Tetralogy of Fallot for Prediction of Remodelling and Therapy Planning
}

Tommaso Mansi ${ }^{1}$, Stanley Durrleman ${ }^{1}$, Boris Bernhardt ${ }^{2}$, Maxime Sermesant ${ }^{1}$, Hervé Delingette ${ }^{1}$, Ingmar Voigt ${ }^{3}$, Philipp Lurz ${ }^{4}$, Andrew M. Taylor ${ }^{4}$, Julie Blanc ${ }^{5}$, Younes Boudjemline ${ }^{5}$, Xavier Pennec ${ }^{1}$, and Nicholas Ayache ${ }^{1}$

1 INRIA-Méditerranée, Asclepios Project, Sophia Antipolis, France

2 Neuroimaging of Epilepsy Laboratory, McGill University, Montreal Neurological Institute, Quebec, Canada

3 Siemens AG, CT SE 5 SCR2, Erlangen, Germany \& Chair of Pattern Recognition, University of Erlangen-Nuremberg, Erlangen, Germany

4 UCL Institute of Child Health \& Great Ormond Street Hospital for Children, London, United Kingdom

${ }^{5}$ Service de Cardiologie Pédiatrique, Hôpital Necker-Enfants Malades, Paris, France

\begin{abstract}
Patients with repaired Tetralogy of Fallot commonly suffer from chronic pulmonary valve regurgitations and extremely dilated right ventricle (RV). To reduce risk factors, new pulmonary valves must be re-implanted. However, establishing the best timing for re-intervention is a clinical challenge because of the large variability in RV shape and in pathology evolution. This study aims at quantifying the regional impacts of growth and regurgitations upon the end-diastolic RV anatomy. The ultimate goal is to determine, among clinical variables, predictors for the shape in order to build a statistical model that predicts RV remodelling. The proposed approach relies on a forward model based on currents and LDDMM algorithm to estimate an unbiased template of 18 patients and the deformations towards each individual shape. Cross-sectional multivariate analyses are carried out to assess the effects of body surface area, tricuspid and transpulmonary valve regurgitations upon the RV shape. The statistically significant deformation modes were found clinically relevant. Canonical correlation analysis yielded a generative model that was successfully tested on two new patients.
\end{abstract}

\section{Introduction}

Tetralogy of Fallot (ToF) is a severe congenital heart defect that requires surgical repair early in infancy. Yet, pulmonary valves may be damaged by the surgery, causing chronic regurgitations. As a result, the right ventricle (RV) dilates extremely, its shape is altered and the cardiac function is impaired: new valves must be implanted in adulthood to reduce risk factors 11. Understanding and quantifying RV remodelling in repaired ToF patients is crucial for patient management and therapy planning. However, high variability in pathology course and in RV anatomy makes difficult the decision of optimal timing for re-intervention [1]. 
Contrary to the left ventricle, whose shape and deformations under pathological conditions are well documented, RV anatomy is complex and can vary tremendously among ToF patients. Several studies investigated possible correlations between clinical parameters in ToF 11. However, few works have quantified the anatomical alterations of the RV and their evolution due to the disease 2213. In 2], the authors measure the most striking differences in RV shape with respect to normals, quantifying some features of the complex RV remodelling observed in ToF. However, only one-dimensional indices are considered despite the availability of 3D segmentations. In [3], the authors present a 4D Active Appearance Model of the beating heart to segment RV in MRI. New indices based on the shape modes are proposed to classify patients from normal. Yet, the authors do not correlate their model with clinical features of ToF.

The clinical challenges raised by ToF encourage applying image-based shape analysis techniques to model the RV anatomical alterations due to pathological factors. These techniques generate a representative template of a population of interest and assess how it deforms within this population [4/5|6/7. Yet, correlating shape with clinical variables require a rigourous framework: Biases may appear if the template is not defined in a consistent way, which may yield drastic differences in the statistical conclusions. Two strategies are available to create the template. The backward approach consists in modelling the template as the average of the deformed observations plus some residuals [45. Such a template can be computed efficiently but the model parameters, especially the residuals, are more difficult to identify. The forward approach consists in modelling the observations as deformations of the template plus some residuals [617. Computing the template is more complex but model parameters can be faithfully estimated from images and clinical data.

In view of assisting the cardiologists in establishing the best time for reintervention, we aim at statistically predict the RV remodelling in ToF. As a first step, we propose in this work to quantify the regional impacts of growth and regurgitations upon the end-diastolic RV anatomy of a cohort of 18 young ToF patients. The main deformation modes are estimated using the forward approach and analysed through cross-sectional multivariate methods. We then derive a generative model of RV remodelling and test it on two new patients.

\section{Methods}

The right ventricle (RV) of multiple patients is segmented from cine-MRI as described in Sec. 3.1. To analyse this population of shapes, an unbiased template is first built. This template serves as reference atlas to determine the deformations towards each individual shape. Then, Principal Component Analysis (PCA) is applied on these deformations to extract the main deformation modes. The importance of each mode is statistically assessed with respect to child growth and valvar regurgitation severity, yielding a generative model of RV remodelling. Finally, we investigate how this model can predict the evolution of shape with respect to body surface area. 


\subsection{Unbiased Template of the Right Ventricle in Tetralogy of Fallot}

The RV template is created using the forward strategy proposed by 7]. This approach is particularly suited for our purposes as 1) it is non-parametric, shapes are represented by currents; 2) model parameters are well-defined and can be estimated from clinical data, thus enabling statistical analyses; 3) template and deformations are computed simultaneously and consistently and 4) new patients can be integrated in the study without re-estimating the template.

The RV surfaces $\mathcal{T}^{i}$, or shapes, are modelled as the sum of a diffeomorphic deformation $\phi^{i}$ of the template $\overline{\mathcal{T}}$ and a residual term $\epsilon^{i}$ standing for the shape features that cannot be represented by the template (topology changes, acquisition artefacts, etc.): $\mathcal{T}^{i}=\phi^{i} \cdot \overline{\mathcal{T}}+\epsilon^{i}$. Currents are used to represent the shapes, the residuals and the deformations in the same common framework. They enable the usual operations (mean, variance...) on shapes as they form a vector space. Intuitively, currents can be seen as the flux of any vector field $\omega \in W$ across the shapes. $W$ is a vector space of infinite dimension generated by a Gaussian kernel $K_{W}(\mathbf{x}, \mathbf{y})=\exp \left(-\|\mathbf{x}-\mathbf{y}\|^{2} / \lambda_{W}^{2}\right)$ that defines an inner product in $W(W$ is a Reproducible Kernel Hilbert Space, RKHS). More precisely, a triangle centred at $\mathbf{x}$ with normal $\alpha$ is represented by the Dirac delta current $\delta_{\mathbf{x}}^{\alpha}$. Therefore, a discrete mesh is encoded by the sum of the currents of its triangles $\mathcal{T}^{i}=\sum_{k} \delta_{\mathbf{x}_{k}^{i}}^{\alpha_{i}^{i}}$. The residuals $\epsilon^{i}$ are modelled as a Gaussian distribution on the $\alpha_{k}^{i}$. The deformation $\phi^{i}$ that registers the template $\overline{\mathcal{T}}$ to the current $\mathcal{T}^{i}$ is estimated using the Large Deformation Diffeomorphic Mappings (LDDMM) framework [8]. $\phi^{i}$ is parametrised by a smooth initial vector speed $\mathbf{v}_{0}^{i}$, which also belongs to a Gaussian RKHS $V$ with variance $\lambda_{V}^{2}$. Moreover, this initial speed vector field is completely defined by the moment vectors $\beta^{i}$ centred at the same point location as the template moments: $\mathbf{v}_{0}^{i}(\mathbf{x})=\sum_{k} K_{V}\left(\mathbf{x}_{k}, \mathbf{x}\right) \beta_{0}^{i}\left(\mathbf{x}_{k}\right)$. Finally, the template $\overline{\mathcal{T}}$ and the deformations $\phi^{i}$ towards each patient are estimated by means of an alternate two-step strategy, initialised with the mean current of the population.

\subsection{Characterising Deformation Modes of RV Shapes in ToF}

In this work we analyse the deformations $\phi^{i}$ only as we mainly focus on the regional changes of the RV anatomy due to ToF. Principal Component Analysis (PCA) is performed directly on the moments $\beta_{i}$ to extract the main deformation modes. The elements of the covariance matrix $\Sigma$ are given by $\Sigma_{i j}=<\mathbf{v}_{0}^{i}-$ $\overline{v_{0}}, \mathbf{v}_{0}^{j}-\overline{v_{0}}>_{V}=\sum_{\mathbf{x}_{k}, \mathbf{x}_{l}}\left(\beta^{i}\left(\mathbf{x}_{k}\right)-\bar{\beta}\left(\mathbf{x}_{k}\right)\right) K_{V}\left(\mathbf{x}_{k}, \mathbf{x}_{l}\right)\left(\beta^{j}\left(\mathbf{x}_{l}\right)-\bar{\beta}\left(\mathbf{x}_{l}\right)\right), \mathbf{x}_{k}$ being the position of the $k^{\text {th }}$ Dirac delta current of $\overline{\mathcal{T}}$. Then, the moment vector $\gamma^{m}$ of the initial speed vector $\mathbf{u}_{0}^{m}$ related to the $\mathrm{m}^{\text {th }}$ deformation mode is given by $\gamma^{m}=$ $\bar{\beta}+\sum_{i} \mathbf{V}^{m}[i]\left(\beta^{i}-\bar{\beta}\right)$. In this equation, $\mathbf{V}_{m}$ is the $\mathrm{m}^{\text {th }}$ eigenvector of $\Sigma$ when the eigenvalues are sorted in decreasing order. Finally, the RV shape of each patient $i$ is characterised by the shape vector $\mathbf{s}^{i}=\left\{s_{m}^{i}\right\}_{m=1 . . M}$ computed from the $M$ first deformation modes, $s_{m}^{i}=<\mathbf{v}_{0}^{i}, \mathbf{u}_{0}^{m}>_{V}=\sum_{\mathbf{x}_{k}, \mathbf{x}_{l}} \beta^{i}\left(\mathbf{x}_{k}\right) K_{V}\left(\mathbf{x}_{k}, \mathbf{x}_{l}\right) \gamma^{m}\left(\mathbf{x}_{l}\right)$. 


\subsection{Can We Predict the Shape from Clinical Parameters?}

First, cross-sectional analysis of the impact of growth on RV shape was performed. Multiple linear regression between the shape vectors $\mathbf{s}^{i}$ and body surface area (BSA) was carried out to exhibit the effects of BSA on each deformation mode. An optimal set of modes was selected by iteratively removing the modes with lowest significance, until the $p$-value of the regression overall significance stopped decreasing. Canonical Correlation Analysis (CCA) was then applied to quantify the amount of variation of each mode when BSA varies. Denoting $R$ the overall correlation coefficient between BSA and shape vectors and $\rho$ the correlation vector relating BSA to each deformation mode, the moments $\mu$ of the generative deformation $\Phi$ are $\mu=R \sum_{k} \rho[k] \gamma^{k}$. Deforming the template $\overline{\mathcal{T}}$ with $\Phi$ enables quantifying the average RV remodelling observed in our population.

Second, we assessed the impact of tricuspid and transpulmonary regurgitations on each deformation mode. As regurgitations were quantified by ordinal indices and only 18 subjects were available, we choose to perform two independent and component-by-component analyses to maximise statistical power. Rank-based Kruskal-Wallis analysis of variance was applied. If an effect was found for some deformation modes, post-hoc two-sample rank-based Wilconxon tests were used to determine which levels differed.

All the statistical tests were carried out using the shape vectors $\mathbf{s}^{i}$ (Sec. 2.2). The level of significance was set at $p<0.1$ and multiple comparisons were corrected using Bonferroni adjustment.

\section{$3 \quad$ Experiments and Results}

\subsection{Data Collection}

Subjects and Image Preparation. We selected 18 young patients (8 males, mean age $\pm \mathrm{SD}=15 \pm 3$ ) with repaired Tetralogy of Fallot (ToF). Body-surface area (BSA) was reported for each patient (Dubois formula, mean $\pm \mathrm{SD}=1.53 \pm$ 0.3 ). Steady-State Free Precession cine MRI of the heart were acquired with a $1.5 \mathrm{~T}$ MR scanner (Avanto, Siemens). Images were acquired in the short-axis view covering entirely both ventricles (10-15 slices; isotropic in-plane resolution: $1.1 \times 1.1 \mathrm{~mm}$ to $1.7 \times 1.7 \mathrm{~mm}$; slice thickness: $6-10 \mathrm{~mm}$; $25-40$ phases). Images were made isotropic through tricubic resampling.

Surface Meshes Preparation. End-diastolic RV endocardium was segmented on the MRI cardiac sequence by fitting an anatomically accurate geometrical model. Its position, orientation and scale in the image was determined using minimal user interaction. Then, local boundaries were estimated by training a probabilistic boosting tree classifier with steerable features [9]. To reduce positioning effects in the shape analysis, the RV meshes were rigidly registered to a representative patient of the dataset by using GMMReg 1 [10. The results were visually inspected and remaining undesirable rotations were corrected manually (Fig. 1, left panel).

${ }^{1}$ http://code.google.com/p/gmmreg/ 


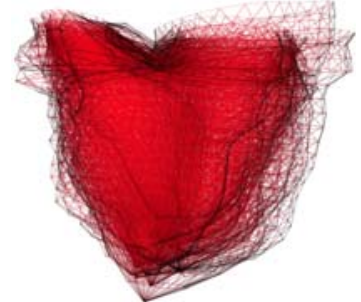

Rigid alignment

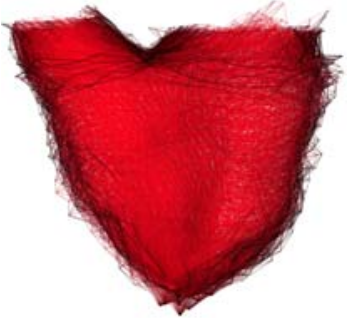

Non-linear registration to the template

Fig. 1. 3D RV meshes of 18 young ToF patients. Left panel: The meshes were rigidly registered to a representative patient of the dataset. Observe the extreme variability in shape (see companion video). Right panel: The same meshes registered to the template using the non-linear deformations estimated during the template creation.

\subsection{Statistical Shape Model of the Right Ventricles}

Building the template $\overline{\mathcal{T}}$ required setting two parameters (Sec. 2.1): $\lambda_{V}$, which defines the "stiffness" of the non-linear deformations (the higher is $\lambda_{V}$, the more global are the transformations); and $\lambda_{W}$, which characterises the resolution of the currents representation (low $\lambda_{W}$ values enable analysing subtle shape features). As we were mainly interested in the regional ToF alterations (dilation, valve enlargement, regional bulging), these parameters were set to $\lambda_{W}=\lambda_{V}=15 \mathrm{~mm}$, about the diameter of the RV outflow tract. Lower values would have been inappropriate as the image slice thickness was approximately $10 \mathrm{~mm}$.

One iteration of the alternate minimisation was needed to reach convergence. Yet, the resulting template $\overline{\mathcal{T}}$ was well centred (mean over standard deviation of the deformations was 0.8 ). The first 10 deformation modes were selected, representing more than $90 \%$ of the total energy (Fig. 2).

Interestingly, the age of the closest patient to the template was 17 while his BSA 1.76. Both indices were close to the observed mean, suggesting that in our population, the mean shape was consistent with the mean BSA and age. Furthermore, this patient only suffers from trace valvar regurgitations, which is not surprising as no evident pathological bulging were visible in the template.

\subsection{Statistical Model of RV Remodelling in ToF Patients}

Patient growth was quantified by body surface area (BSA) index (correlation with age in the data set: $\left.R^{2}>0.5, p<0.001\right)$. Table 1 reports the regression coefficients $a_{l}$ of the multiple linear regression between BSA and shape vectors $\mathbf{s}$, $\mathrm{BSA}=a_{0}+\sum_{l=1}^{10} a_{l} \mathbf{s}[l]$, the related $t$-values and the overall model significance. The sign of the $a_{l}$ relates to the direction of the deformation modes (negative $a_{l}$ meaning backwards deformations). Model reduction discarded all the nonsignificant modes (Table1). The remaining modes were found clinically pertinent by an expert after visual inspection (Fig. 2). Mode 1 clearly represented the overall RV dilation. Mode 2 seemed to model the dilation of the tricuspid annulus and of the inflow tract. Mode 3, 6, 7 and 9 exhibited a dilation of a specific RV 


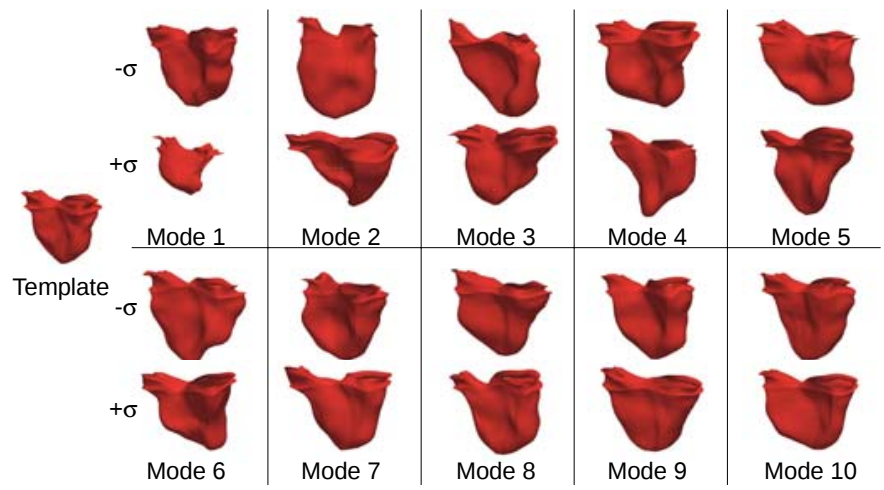

Fig. 2. 10 first deformation modes extracted by PCA on a population of 18 patients suffering from repaired Tetralogy of Fallot

Table 1. Linear regression coefficients $a_{l}$ between shape modes and BSA. In bold the significant coefficients $(p<0.1)$. After model reduction (second array), coefficients stay unchanged, confirming the stability of the statistical tests.

\begin{tabular}{|c|c|c|c|c|c|c|c|c|c|c|c|}
\hline & Significance & $\mathbf{a}_{1}$ & $a_{2}$ & $a_{3}$ & $a_{4}$ & $a_{5}$ & $a_{6}$ & $a_{7}$ & $a_{8}$ & $a_{9}$ & $a_{10}$ \\
\hline Coef. $\times 10^{-}$ & \multirow{2}{*}{$\begin{array}{c}R^{2}=0.84 \\
p=0.04\end{array}$} & -2.9 & 6.4 & -7.6 & 4.6 & -1.0 & -11.1 & $\mid-11.9$ & 7.0 & -20.1 & -15.4 \\
\hline$t$-values & & -3.28 & 2.64 & -2.04 & 1.13 & -0.19 & -1.93 & -1.92 & 0.84 & -2.15 & -1.43 \\
\hline Coef. $\times 10^{-5}$ & \multirow{2}{*}{$\begin{array}{l}R^{2}=0.75 \\
p=0.006\end{array}$} & -2.9 & 6.4 & -7.6 & & & -11.1 & -11.9 & & -20.0 & \\
\hline$t$-values & & -3.27 & 2.63 & -2.03 & & & -1.92 & -1.92 & & -2.14 & \\
\hline
\end{tabular}

region: apex (mode 3), basal area under the tricuspid valve (mode 6), apical area of the outflow tract (mode 7 ) and outflow tract (mode 9), reflecting possible direct impact of regurgitations on the neighbouring tissues.

Canonical Correlation Analysis (CCA) provided a generative model of the RV remodelling observed in our population. Overall correlation coefficient with BSA was $R=0.87$, suggesting a strong correlation between these deformation modes and growth. The correlation vector of the deformation modes was $\rho=\{-0.56,0.45,-0.35,-0.33,-0.33,-0.37\}$. When BSA increases by 0.86 , each deformation mode $m$ varies by its related coefficient $\rho[m]$. The model was found clinically realistic by an expert (Fig. 3). As BSA increased, RV volume increased, RV free-wall and valves dilated, and septum was more concave. Indeed, dilation of the valves reduces the remaining pulmonary obstructions, thus decreasing the RV pressure. As a result, left-ventricle pushes the septum towards the right ventricle, making it more concave. However, as regurgitations are still present, the RV still dilates by pushing the RV free wall outwards.

\subsection{Quantifying the Impact of Valvar Regurgitations on RV Shape}

Colour Doppler ultrasound (sweep speeds: 50-100 mm/s) was used to quantify tricuspid (TriReg) and transpulmonary valve (TPVReg) regurgitations. To assess the effects of TPVReg, patients were grouped into two different groups: 


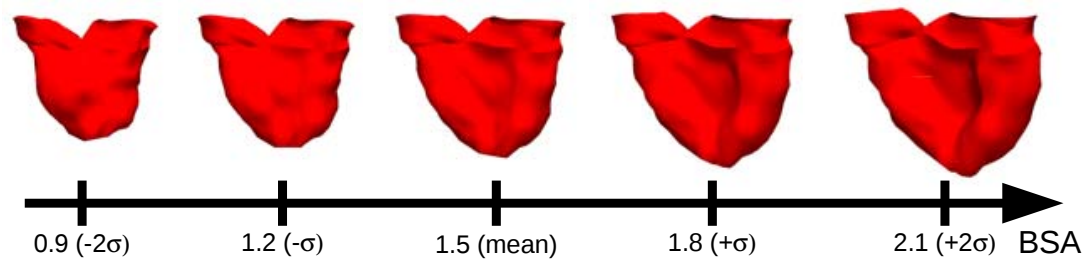

Fig. 3. Mean RV remodelling observed in our population when body surface area (BSA, in $\mathrm{m}^{2}$ ) increases. RV dimensions globally increase while valves dilate. Simultaneously, $\mathrm{RV}$ free wall becomes rounder and septum more concave (see companion videos).

trace TPVReg and severe TPVReg. Kruskal-Wallis analysis revealed a significant effect on deformation mode $2(p<0.1)$, which was confirmed by visual inspection: this mode exhibited an elongation of the RV outflow tract (Fig. 2).

Evaluation of TriReg classified the patients into 3 groups: none, trace or mild tricuspid regurgitations. Kruskal-Wallis analysis showed a significant impact of TriReg on three deformation modes: $3(p<0.05), 6(p<0.1)$ and $8(p<0.1)$. However, pair-wise Wilconxon tests showed that only mode 3 had two significant different levels (trace TriReg versus mild TriReg, $p<0.1$ ). Visually, deformation mode 3 exhibited a deformation of the tricuspid annulus, from circular to triangular-shape, and a dilation of the RV inflow tract.

Interestingly, two deformation modes involved in the statistical model of RV growth were also related to the regurgitations. This may suggest possible crosseffects between growth and regurgitations on these specific shape variations.

\subsection{Validating the Generalisation of the Statistical Models}

Generalising a statistical model of RV remodelling is crucial for patient management and therapy planning. We thus tested the robustness of our model on two new patients with matched age (13 and 16). The template was registered to the patients and the related shape vectors $\mathbf{s}$ were computed. BSA were calculated from the optimal linear model estimated in Sec. 3.3. Results successfully compared with measured values (patient 1: estimated BSA: 1.61, measured BSA: 1.49; patient 2: estimated BSA: 1.29, measured BSA: 1.16). This suggests that the deformation modes involved in the RV remodelling model could be generalised, constituting potential quantitative parameters of remodelling in ToF.

\section{Discussion and Future Works}

In this study we investigated the impact of growth and valvar regurgitations upon the end-diastolic RV anatomy of patients with repaired ToF. End-diastolic time point was chosen as it is the time when the effects of the pathology are the most evident 12. Multivariate statistical analyses provided a generative model of the observed RV remodelling. This model and the significant deformation modes were found clinically pertinent as they exhibited remarkably realistic changes in RV anatomy. To design the model, the deformation modes and their 
directions were statistically determined to limit the effects of PCA rotatability. Furthermore, the effects of regurgitation severity were analysed on a componentby-component basis to preserve the statistical power of the tests due to the ordinal nature of the data. The groups were not sufficiently populated to apply more comprehensive statistics. Incorporating more patients is now required to confirm these findings and avoid possible over-interpretations. Various types of RV remodelling could be identified (aneurysmal, with stiff myocardium, etc.), which may constitute new criteria for valve replacement decision. Future works also include analysing the 4D cardiac motion. To the best of our knowledge, this study constitutes a first attempt at correlating 3D shape parameters to clinical measurements in ToF. These analyses may yield quantitative image-based predictors about RV anatomy and remodelling in ToF.

Acknowledgments. This work has been partly funded by the European Commission, IST-2004-027749 Health-e-Child Integrated Project. The authors warmly thank Pr. G. Pongiglione, Istituto G. Gaslini, Genova, for his helpful comments about the models.

\section{References}

1. Geva, T.: Indications and timing of pulmonary valve replacement after tetralogy of Fallot repair. In: Seminars in Thoracic and Cardiovascular Surgery: Pediatric Cardiac Surgery Annual, vol. 9, pp. 11-22. Elsevier, Amsterdam (2006)

2. Sheehan, F., Ge, S., Vick III, G., Urnes, K., Kerwin, W., Bolson, E., Chung, T., Kovalchin, J., Sahn, D., Jerosch-Herold, M., et al.: Three-Dimensional Shape Analysis of Right Ventricular Remodeling in Repaired Tetralogy of Fallot. The American Journal of Cardiology 101(1), 107 (2008)

3. Zhang, H., Walker, N., Mitchell, S., Thomas, M., Wahle, A., Scholz, T., Sonka, M.: Analysis of four-dimensional cardiac ventricular magnetic resonance images using statistical models of ventricular shape and cardiac motion. In: Proc. SPIE 2006, vol. 6143 , p. $614307(2006)$

4. Guimond, A., Meunier, J., Thirion, J.P.: Average brain models: A convergence study. Computer Vision and Image Understanding 77(2), 192-210 (2000)

5. Joshi, S., Davis, B., Jomier, M., Gerig, G.: Unbiased diffeomorphic atlas construction for computational anatomy. NeuroImage 23, 151-160 (2004)

6. Allassonniere, S., Amit, Y., Trouve, A.: Towards a coherent statistical framework for dense deformable template estimation. Journal of the Royal Statistical Society: Series B (Statistical Methodology) 69(1), 3-29 (2007)

7. Durrleman, S., Pennec, X., Trouvé, A., Ayache, N.: A forward model to build unbiased atlases from curves and surfaces. In: Pennec, X., Joshi, S. (eds.) Proc. of the International Workshop on the Mathematical Foundations of Computational Anatomy, MFCA 2008 (2008)

8. Vaillant, M., Glaunès, J.: Surface matching via currents. In: Christensen, G.E., Sonka, M. (eds.) IPMI 2005. LNCS, vol. 3565, pp. 381-392. Springer, Heidelberg (2005)

9. Zheng, Y., Barbu, A., Georgescu, B., Scheuering, M., Comaniciu, D.: Fast automatic heart chamber segmentation from 3D CT data using marginal space learning and steerable features. In: Proc. ICCV 2007, pp. 1-8 (2007)

10. Jian, B., Vemuri, B.: A robust algorithm for point set registration using mixture of Gaussians. In: Proc. ICCV 2005, vol. 2, pp. 1246-1251 (2005) 